\section{Donor-recipient gender difference affects severity of dry eye after hematopoietic stem cell transplantation}

\begin{abstract}
Purpose To determine whether the incidence rate and severity of dry eye after hematopoietic stem cell transplantation varies with donor $v s$ recipient gender.

Methods We limited this study to patients received bone marrow transplantation (BMT). In all, 172 patients received BMT at Keio University School of Medicine between January 2000 and May 2007. Of them, 136 recipients who survived at least 70 days were studied prospectively. We classified the 136 patients according to the gender of the donor and the recipient (group I: female to female; group II: male to male; group III: male to female; group IV: female to male). The incidence and severity of chronic graft-vs-host disease-associated dry eye were determined for each group. The donor gender was masked when we assessed dry eye and calculate the incidence.
\end{abstract}

Results The incidence of dry eye was $47.4 \%$ for group I, $37.5 \%$ for group II, $58.6 \%$ for group III, and $42.9 \%$ for group IV. The percentage of patients with severe dry eye was $44.4,50.0$, 35.3 , and $77.8 \%$ respectively. There was a significant difference between the percent severe dry eye/total dry eye incidences in groups III and IV ( $P=0.0375)$ (odds ratio, 7.6; 95\% confidence interval, 1.00-101.01).

Conclusions Close attention must be paid to the development of dry eye in cases of female to male BMTs, because the ratio of severe/total dry eye is more common in cases of female to male BMTs than in other gender combination. Eye (2011) 25, 860-865; doi:10.1038/eye.2011.73; published online 8 April 2011

Received: 6 September 201 Accepted in revised form: 11 February 2011 Published online: 8 April 2011

Keywords: dry eye; allogeneic hematopoietic
M Kamoi', Y Ogawa', M Uchino', Y Tatematsu', T Mori $^{2}$, S Okamoto ${ }^{2}$ and K Tsubota ${ }^{1}$

disease; gender-mismatched transplantation; minor histocompatibility antigens

\section{Introduction}

Graft-vs-host disease (GVHD) is a major complication subsequent to hematopoietic stem cell transplantation (HSCT). Many HSCT recipients become long-term survivors of their disease, and chronic GVHD (cGVHD) occurs in $30-70 \%$ of allogeneic HSCT recipients. ${ }^{1,2}$ As a result of this, assuring patient quality of life (QOL) and addressing late complications after HSCT have become increasingly important. The eye, mouth, liver, lung, skin, and intestine are preferential targets of cGVHD. ${ }^{3}$ Dry eye has emerged as a major complication of systemic cGVHD as well as ocular cGVHD that strongly affects patient QOL. ${ }^{4}$ So far, neither radical treatment nor prophylaxis has been established for cGVHD-related dry eye. This study was intended to learn which patients are at the higher risk for developing progressive dry eye.

It is generally known that a donor-recipient gender mismatch in HCST often leads to severe GVHD. ${ }^{5}$ In particular, GVHD in male recipients of female donors tends to be especially severe. ${ }^{6-9}$ Given the current knowledge about gender mismatch and GVHD, it seems likely that HSCT with a female donor and male recipient could increase the patient's risk of developing cGVHD-related severe dry eye. However, no report on donor-recipient gender mismatch affecting ocular GVHD has been published in the ophthalmologic literature.

In this retrospective study, we focused on stem cell transplantation; chronic graft-vs-host donor-recipient gender only in cases treated by bone marrow transplantation (BMT), and 
investigated the incidence and severity of dry eye associated with matched and mismatched gender in donor-recipient pairs.

\section{Patients and methods}

\section{Study design}

This was a retrospective survey evaluating the late effects of BMT. All research and measurements followed the tenets of the Declaration of Helsinki. The study was approved by the ethics committee of the Keio University School of Medicine. All patients underwent standardized clinical and ophthalmological evaluation, as described below, before BMT and 3, 6, 9, 12, 18, 24, and 30 months after BMT, as well as on additional occasions as indicated.

Between January 2000 and May 2007, 172 patients underwent BMT at Keio University School of Medicine. We selected patients who survived for at least 70 days after BMT for this study. This criterion selected patients who survived beyond the time of acute GVHD (aGVHD) development and who could potentially develop cGVHD. We excluded patients under the age of 15 and those who underwent re-transplantation. In total, 36 of the 172 recipients were excluded from this study, and the remaining 136 patients were studied prospectively. When we examined BMT patients, the donor genders were masked. We classified the 136 patients according to the genders of the donor and recipient, and determined the incidence rate and severity of dry eye among these groups ((donor to recipient) group I: female to female; group II: male to male; group III: male to female; group IV: female to male).

\section{Clinical evaluation}

Ocular surface vital staining

The fluorescein and rose bengal stain scores for the ocular surface were obtained using the double-vital staining method. ${ }^{10-13}$ Two microliter of a preservativefree solution of $1 \%$ rose bengal and $1 \%$ fluorescein was instilled into the conjunctival sac by a micropipette. ${ }^{10}$ The van Bijsterveld scoring system was used for the rose bengal staining. ${ }^{14}$ For the rose bengal score, the ocular surface was divided into the three zones: nasal conjunctival, corneal, and temporal. A score of $0-3$ points was used for each zone, with a minimum possible score of 0 and a maximum possible score of 9 . Scarce punctuate staining was given 1 point. Denser staining not covering the entire zone was given 2 points. Denser staining over the entire zone was given 3 points. For fluorescein staining, the cornea was divided three equal upper, middle, and lower zone. Each zone has a staining score ranging from 0 to 3 points, as with the rose bengal stain, and a minimum and maximum score was 0 and 9 , respectively. The presence of scarce staining in a zone was scored 1; frequent puncta not covering the entire zone was scored as 2 points; and puntate staining covering the entire zone was scored as 3 points. ${ }^{15}$

\section{Tear function test}

Tear film break-up time (TBUT) was measured three times at the time of double staining, and the median value was calculated. ${ }^{11}$ The Schirmer test was performed using standard strips (Alcon, Fort Worth, TX, USA) placed in the lower conjunctival sac for $5 \mathrm{~min}$ without anesthesia. For the Schirmer test with nasal stimulation, the standard strips were placed in the conjunctival sac for $5 \mathrm{~min}$, while a cotton-wool swab was inserted into the nose until the tip reached the nasal membrane of the ethmoid sinus. The middle turbinate was touched with the cotton-wool swab, which was kept in place for $5 \mathrm{~min}$. We then measured the length of the moistened part of the standard strip from the conjunctival sac. ${ }^{16}$

\section{Diagnostic criteria}

Dry eye was diagnosed as a disorder of tear film caused by tear deficiency and/or excessive tear evaporation, which cause the damage of ocular surface with or without symptom. ${ }^{12}$ Dry eye was diagnosed when the tear film of patients showed disturbance of tear dynamics (TBUT $\leq 5 \mathrm{~s}$, Schirmer test $\leq 5 \mathrm{~mm}$ ) and the ocular surface was abnormal (rose bengal score $\geq 3$, fluorescein score $\geq 1) .{ }^{13}$ Severe dry eye was defined as reduced reflex tearing (Schirmer test with nasal stimulation $\leq 10 \mathrm{~mm}$ ) and abnormality of the ocular surface (rose bengal score $\geq 3$ and/or fluorescein score $\geq 3)^{14}$ and/or a grade of 3 or 4 according to the dry eye workshop (DEWS) report $2007 .{ }^{17}$ Briefly, DEWS proposed the classification of dry eye severity level, which is classified according to the grade of symptom, ocular surface findings, and tear dynamics. The severity level 3 or 4 was regarded as the sign of severe frequent or constant and/or disabling symptom, severe ocular surface damage accompanied by marked injection, filamentary keratitis, mucous clumping, tear debris, and ulceration. When trichiasis, keratinization, and symblepharon along with sign of symptom of dry eye were present, dry eye was also regarded as grade 3 and 4 . In addition, TBUT and Schirmer scores are $\leq 5 \mathrm{~s}$ and $\leq 5 \mathrm{~mm}$ for grade 3 and immediate and $\leq 2 \mathrm{~mm}$ for grade 4 , respectively.

Mild dry eye was defined as abnormality of the ocular surface (rose bengal score $\geq 3$, fluorescein score $\geq 1$ ) without reduced reflex tearing (Schirmer test with nasal stimulation $>10 \mathrm{~mm}$ ). According to the grading system of the severity level of dry eye based on DEWS report, 
grade 1 and 2 were regarded as mild to moderate stress, and none to mild, or variable conjuntival injection, conjunctival or corneal staining or corneal/tear signs. In addition, TBUT and Schirmer scores are variable for grade 1 , and $\leq 10$ s and $\leq 10 \mathrm{~mm}$ for grade 2 , respectively. Patients who had dry eye before BMT were considered to have a sustained a dry eye incident only when the severity of the dry eye worsened after the transplantation.

\section{Statistical analysis}

Fisher's direct method was used to evaluate differences among the groups. Statistical analyses were performed using $R$ statistical software (Free Software Foundation, Boston, MA, USA). $R$ is available freely on web site. This is an open source software for statistical analysis. ${ }^{18,19}$

In this article, version-2.9.1 was available. Significant difference was defined as $P \leq 0.05$.

\section{Results}

The 136 subjects survived for at least 70 days after BMT and were evaluable for the presence and severity of dry eye. The median age of the patients was 44 years (range 18-61 years). The median age of the donors was 35 years (range 18-80 years). Clinical characteristics of the 136 patients are shown in Table 1 . There were no significant differences between patient and donor age in any group. The percentage of unrelated donors was higher than that of related donors in all groups. There was no statistical significant when we carried out a statistical analysis concerning related/unrelated parameters of donor, that is, the genetic influence in all four groups (Table 1).

We showed the baseline profile of pre-BMT and the serial change of ophthalmic findings at pre- and postBMT (Tables 2 and 3, Figure 1). There were significant differences of the clinical variables of ocular surface findings and tear dynamics between at the pre- and post-BMT (Figure 1).

Table 1 Patient characteristics in the four donor-recipient gender groups

\begin{tabular}{lcccc}
\hline & $\begin{array}{c}I(F \rightarrow F) \\
\mathrm{n}=38\end{array}$ & $\begin{array}{c}I I(M \rightarrow M) \\
\mathrm{n}=48\end{array}$ & $\begin{array}{c}I I I(M \rightarrow F) \\
\mathrm{n}=29\end{array}$ & $\begin{array}{c}I V(F \rightarrow M) \\
\mathrm{n}=21\end{array}$ \\
\hline $\begin{array}{l}\text { Patient age (range) } \\
\text { Donor age (range) }\end{array}$ & $39.5(20-60)$ & $44.5(18-59)$ & $43(25-61)$ & $47(18-59)$ \\
& $36(33-63)$ & $33(17-58)$ & $38(21-80)$ & $36(23-52)$ \\
$\begin{array}{l}\text { Donor relation } \\
\text { Unrelated (\%) }\end{array}$ & $27(71)$ & $36(75)$ & $23(79.3)$ & $17(81)$ \\
$\quad$ Related (\%) & $11(29)$ & $12(25)$ & $6(20.7)$ & $4(19)$ \\
\hline
\end{tabular}

Abbreviations: $\mathrm{F}$, female; $\mathrm{M}$, male.
The incidence rate of mild dry eye, severe dry eye, and total dry eye was not significantly different in any of the four groups (Table 4). However, the percentage of patients with dry eye that became severe was greatest in group IV $(77.8 \%)$, and the incidence was significantly higher than in group III $(35.3 \%)(P=0.0375)$. Odds ratio (OR) was 7.6 (95\% confidence interval: 1.00-101.01). The severity of dry eye was more prevalent in group IV more than group III (Table 4, Figure 2). We double checked the severity of dry eye using diagnostic criteria 2007, but the severity determinations were the same for our usual criteria $^{12}$ and the DEWS criteria. ${ }^{17}$

We then evaluated the results by pooling the two gender-matched groups and the two gender-mismatched ones. The incidence rate for total, mild, and severe dry eye was slightly higher in the gender-mismatched group, although significant differences were not observed between these two groups (Figure 3).

\section{Discussion}

Here, we investigated the incidence rate and severity of dry eye associated with matched and mismatched genders in donors and recipients after HSCT, in patient groups with few differences in patient characteristics (Table 1).

The efficacy of HSCT is improved by the graft-vsleukemia (GVL) effect, a result of moderate aGVHD, but the downside of the GVL effect is an increased risk of cGVHD. Therefore, it is important to control the degree of GVHD. It is known that host rejection and GVHD occur even in HLA-matched donor-recipient pairs, because of mismatches in the minor histocompatibility antigens. ${ }^{6,8,9}$

We found that, if dry eye occurred at all, it usually became severe among male BMT recipients whose donor was female (OR, 7.6). Conversely, there was much less progression to severe dry eye in female BMT recipients of donor tissue from males. These findings are consistent with previous reports on the severity of systemic GVHD, in which male HSCT recipients of female donor tissue had a significantly higher probability of developing systemic GVHD compared with the recipients of other recipient-donor gender combinations. ${ }^{6-9}$ These results indicate that dry eye well reflects systemic cGVHD, suggesting that dry eye could be a hallmark of cGVHD based on the assessment of dry eye parameters compared with pre-BMT (Figure 1). There was no difference in the overall incidence of dry eye among the four groups. However, there was a significant difference in the percentage of severe dry eye/total dry eye between the two gender mismatched groups, suggesting that gender-related factors affect the severity of dry eye after 
Table 2 Baseline profile ocular surface findings and tear dynamics of pre-BMT

\begin{tabular}{lccccc}
\hline Pre-F & RB & TBUT & $S$ & $S(N)$ & $M G D$ \\
\hline $0.49 \pm 1.09(n=136)$ & $0.19 \pm 1.07(n=135)$ & $9.36 \pm 1.05(n=117)$ & $12.95 \pm 1.72(n=129)$ & $19.02 \pm 10.31(n=84)$ & $0.86 \pm 1.18(n=40)$
\end{tabular}

Abbreviations: F, fluorescein score; MGD, meibomian gland dysfunction; RB, rose bengal score; S, value of Schirmer test; $\mathrm{S}$ (N), value of Schirmer test with nasal stimulation; TBUT, tear film break-up time.

Table 3 Summary of clinical evaluation at pre- and post-BMT

\begin{tabular}{|c|c|c|c|c|c|c|}
\hline & $F$ & $R B$ & TBUT & $S$ & $S(N)$ & $M G D$ \\
\hline \multicolumn{7}{|l|}{ Pre } \\
\hline $\mathrm{I}(n=38)$ & $0.5 \pm 0.9(38)$ & $0.1 \pm 0.5$ & $9.0 \pm 2.1$ & $14 \pm 11.8$ & $18.0 \pm 10.4$ & $0.8 \pm 1.1$ \\
\hline II $(n=48)$ & $0.5 \pm 1.2$ & $0.1 \pm 0.5$ & $9.7 \pm 1.1$ & $11.9 \pm 10.5$ & $16.1 \pm 9.0$ & $0.9 \pm 1.3$ \\
\hline III $(n=29)$ & $0.3 \pm 0.7$ & $0.1 \pm 0.6$ & $9.6 \pm 1.1(27)$ & $11.8 \pm 8.5(29)$ & $20.4 \pm 11.2$ & $0.8 \pm 0.7$ \\
\hline IV $(n=21)$ & $0.8 \pm 1.5$ & $0.4 \pm 0.9$ & $9.2 \pm 1.6(18)$ & $14.2 \pm 12.0$ & $21.6 \pm 11.3(10)$ & $1.0 \pm 1.3$ \\
\hline \multicolumn{7}{|l|}{ Post } \\
\hline $\mathrm{I}(n=38)$ & $2.1 \pm 2.6$ & $1.7 \pm 2.2$ & $5.7 \pm 3.5$ & $5.6 \pm 7.9$ (17) & $6.4 \pm 5.6(9)$ & $1.9 \pm 1.0(12)$ \\
\hline II $(n=48)$ & $2.1 \pm 2.4$ & $1.6 \pm 2.3$ & $7.0 \pm 3.1(46)$ & $6.8 \pm 5.2(18)$ & $12.1 \pm 8.6$ & $1.7 \pm 0.9$ (9) \\
\hline III $(n=29)$ & $2.1 \pm 2.1$ & $1.5 \pm 2.1$ & $6.2 \pm 3.3(27)$ & $6.1 \pm 6.0$ & $8.9 \pm 7.6(7)$ & $2.3 \pm 0.7(7)$ \\
\hline IV $(n=21)$ & $2.0 \pm 2.1$ & $2.0 \pm 2.2(20)$ & $5.9 \pm 3.2(21)$ & $6.9 \pm 7.5$ & $9.4 \pm 6.5(10)$ & $3.0 \pm 0.0$ \\
\hline
\end{tabular}

Abbreviations: F, fluorescein score; MGD, meibomian gland dysfunction; Pre, pre-BMT; Post, post-BMT; RB, rose bengal score; S, value of Schirmer test; $\mathrm{S}(\mathrm{N})$, value of Schirmer test with nasal stimulation; TBUT, tear film break-up time.

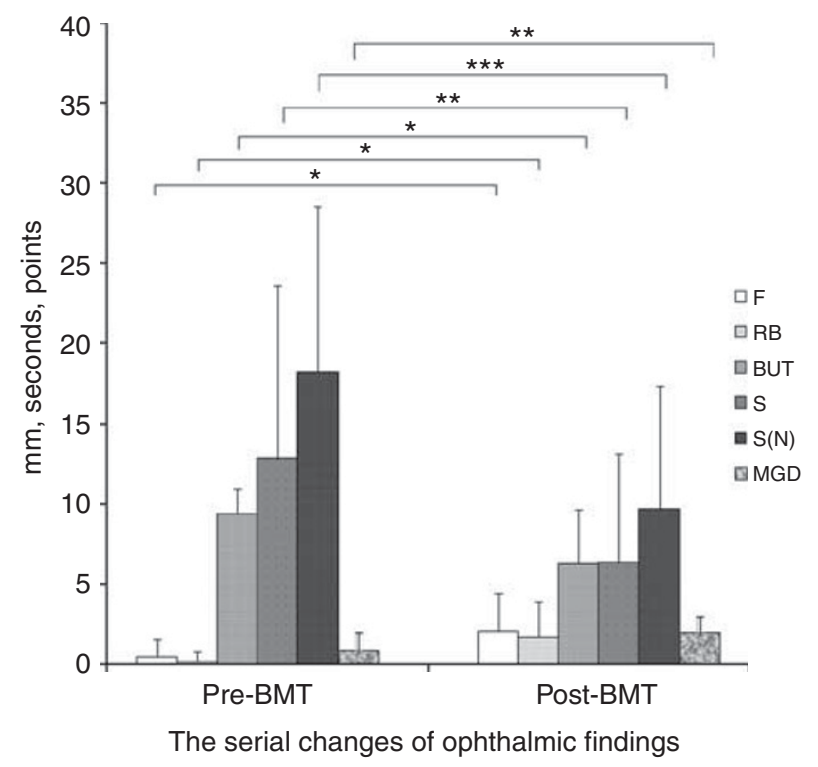

Figure 1 Clinical variables for dry eye parameters at pre- and post-BMT. There were significant differences in ophthalmic findings of pre- and post-BMT. Ocular surface findings and tear dynamics were deteriorated after BMT. F, fluorescein score; RB, rose bengal score; TBUT, tear film break-up time; $S$, value of Schirmer test; $\mathrm{S}(\mathrm{N})$, value of Schirmer test with nasal stimulation; MGD, meibomian gland dysfunction. ${ }^{*} P<0.001,{ }^{* *} P<0.01$, $* * * P<0.05$.

BMT (Figure 2). Particularly, male BMT recipients from female donors have possibility of progressing of severer dry eye.
Table 4 Incidence rate of dry eye, mild dry eye, severe dry eye, and severe/total dry eye in the four groups

\begin{tabular}{lcccc}
\hline & $I$ & $I I$ & $I I I$ & $I V$ \\
\hline Mild type (\%) & 26.3 & 18.8 & 37.9 & 9.5 \\
Severe type (\%) & 21.1 & 18.8 & 20.7 & 33.3 \\
Total dry eye (\%) & 47.4 & 37.5 & 58.6 & 42.9 \\
Severe/total dry eye (\%) & 44.4 & 50 & 35.3 & $77.8^{*}$ \\
\hline
\end{tabular}

${ }^{*} P=0.0375$

Copelan ${ }^{20}$ reported that minor antigens encoded by genes on the $\mathrm{Y}$ chromosome account for the higher incidence of GVHD and lower rate of patient relapse among male recipients of marrow transplants from female donors than among male recipients of transplants from male donors. Miklos et al proposed that, because female donor $\mathrm{T}$ cells have not been exposed to unique epitopes on the $Y$ chromosome, thymic maturation does not delete the $\mathrm{T}$ cells capable of recognizing the $\mathrm{H}-\mathrm{Y}$ antigens. ${ }^{21}$ Besides the T cells, antibodies against the $\mathrm{H}-\mathrm{Y}$ antigen made by donor B cells probably also contribute to GVHD. ${ }^{21,22}$ There are some reports that male recipients whose female donors had previous pregnancies or blood transfusions are at increased risk of developing GVHD, ${ }^{23-25}$ apparently because of B-cell sensitization to the H-Y antigen. In this study, the previous donor pregnancies and transfusions was not checked. Although many factors contribute to the severity, there is a possible that our study supports these earlier findings, showing a higher percentage of cGVHD-associated dry eye 
progressing to the severe form in male recipients of bone marrow from a female donor.

We also pooled the groups to compare all gendermatched pairs with all gender-mismatched pairs. We found the incidence of dry eye in the gender-mismatched pairs to be slightly higher than in gender-matched pairs, although the difference was not significant (Figure 3). This finding also agrees with previous studies in the medical literature, in which the occurrence of GVHD in gender-mismatched pairs was higher than in gender-matched pairs. ${ }^{5}$ However, some other reports have not shown a significant difference in GVHD incidence between gender-mismatched and gendermatched pairs. ${ }^{24,26}$ In this study, we found no significant differences in patient age in these two groups, ruling out age as a contributing factor. However, the diagnosis, stage of the disease, and genetic relatedness between the donor and the recipient, which we did not take into account, all seem to affect the incidence of GVHD.

The only significant difference was the percentage of patients with dry eye who developed severe dry eye, and this difference reached significance only in the group III vs group IV comparison. However, patient refusal of the Schirmer test with nasal stimulation may have distorted the data from this test (Table 3). We therefore, double checked the severity level of dry eye according to the DEWS report 2007.

Our study indicates that there was a significant association between severe dry eye and female to male

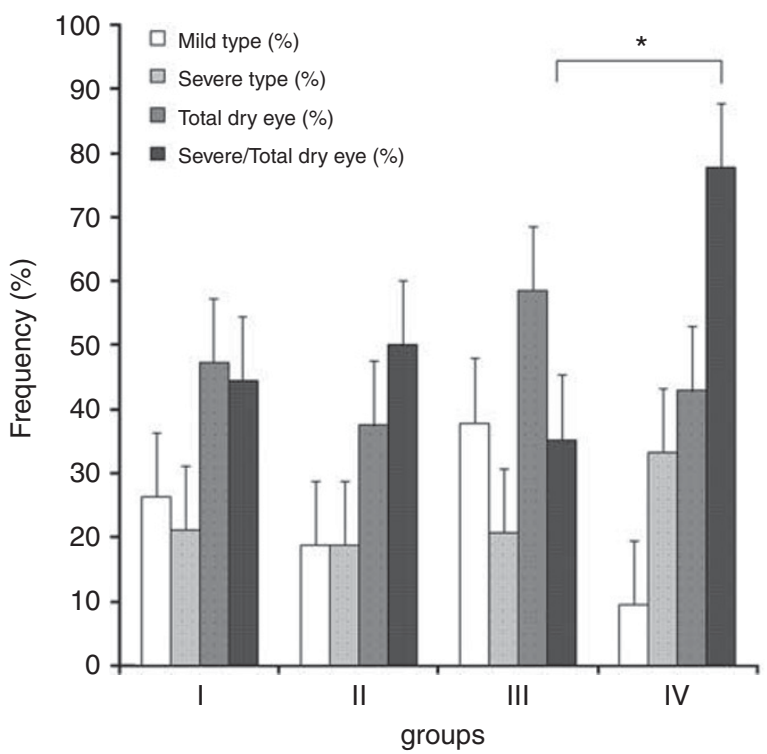

Figure 2 Comparison of the frequency of various type dry eye among four groups. The patients with dry eye that became severe was greatest in group IV $(77.8 \%)$, and the incidence was significantly higher than in group III $(35.3 \%)(* P=0.0375)$. OR was 7.6 (95\% confidence interval: 1.00-101.01). The severity of dry eye was more prevalent in group IV more than group III.
BMT. We are unaware of any previous report on the severity of dry eye correlating with a gender-donor mismatch, although we searched PubMed and MEDLINE reviews. Until now, little attention has been paid to the possible risk for severe dry eye after HSCT associated with donor-recipient gender mismatch. Our study indicates that careful observation is needed for male BMT recipients of female donors. As dry eye well reflects systemic cGVHD, suggesting that dry eye could be a hallmark of cGVHD. As severe dry eye can lead to blindness and poor QOL, early detection to prevent dry eye progression is important.

In this study, we investigated the effect of donor-recipient mismatch on dry eye and severe dry eye incidence, without taking into consideration the relatedness of donor and recipient, previous donor pregnancies, or the recipient's disease diagnosis or stage. Further study will be required to understand the relative impact of these various factors, and will lead to a better understanding of cGVHD.

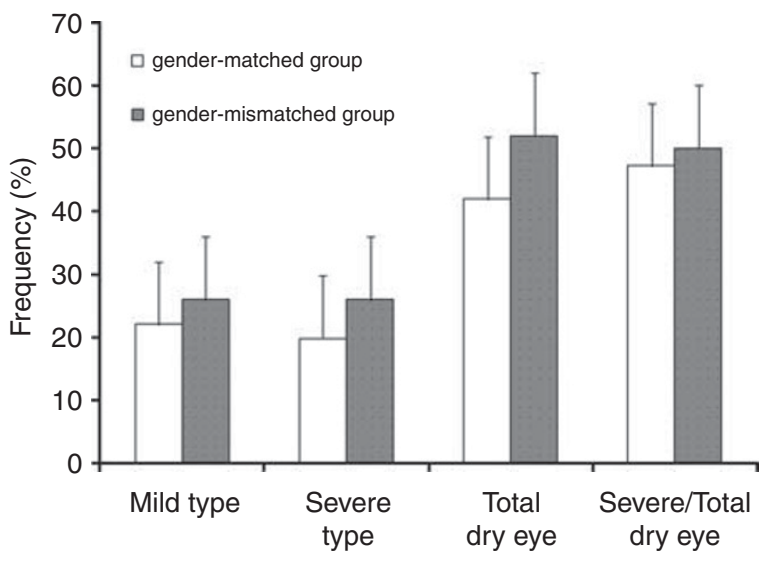

The clinical characteristic of the 2 groups

Figure 3 Comparison of the frequency of various type dry eye between gender-matched and gender-mismatched groups. There were no significant differences between gender-matched and gender-mismatched group. However the incidence rate of total, mild, and severe dry eye was slightly higher in gendermismatch group.

\section{Summary}

What was known before

- It is generally known that a donor-recipient gender mismatch in HCST often leads to severe systemic GVHD. In particular, GVHD in male recipients of female donors tends to be especially severe.

What this study adds

- So far, no report on donor-recipient gender mismatch affecting ocular GVHD has been published in the ophthalmologic literature. Our study indicates that there was a significant association between severe dry eye and female to male BMT. 


\section{Conflict of interest}

The authors declare no conflict of interest.

\section{Acknowledgements}

This study was supported in part by grants from the Japanese Ministry of Education, Science, Sports, and Culture (no. 20592058).

\section{References}

1 Arai S, Vogelsang GB. Management of graft-versus-host disease. Blood Rev 2000; 14: 190-204.

2 Nash RA, Antin JH, Karanes C, Fay JW, Avalos BR, Yeager AM et al. Phase 3 study comparing methotrexate and tacrolimus with methotrexate and cyclosporine for prophylaxis of acute graft-versus-host disease after marrow transplantation from unrelated donors. Blood 2000; 96: 2062-2068.

3 Sullivan KM. Graft-vs-host disease. In: Blume KG, Forman SJ, Applebaum FR (eds). Thomas' Hematopoietic Cell Transplantation. 3rd edn. Blackwell: Malden, MA, 2004, pp 635-664.

4 Ogawa Y, Kuwana M. Dry eye as a major complication associated with chronic graft-versus-host disease after hematopoietic stem cell transplantation. Cornea 2003; 22: S19-S27.

5 Bross DS, Tutschka PJ, Farmer ER, Beschorner WE, Braine HG, Mellitis ED et al. Predictive factors for acute graft-versus-host disease in patients transplanted with HLA-identical bone marrow. Blood 1984; 63: 1265-1270.

6 Randolph SSB, Gooley TA, Warren EH, Appelbaum FR, Riddell SR. Female donors contribute to a selective graftversus-leukemia effect in male recipients of HLA-matched, related hematopoietic stem cell transplants. Blood 2004; 103 347-352.

7 Gale RP, Bortin MM, Bekkum DW, Biggs JC, Dicke KA, Gluckman E et al. Risk factors for acute graft-versus-host disease. Br J Haematol 1987; 67: 397-406.

8 Stern M, Brand R, Witte T, Sureda A, Rocha V, Passweg J et al. Female-versus-male alloreactivity as a model for minor histocompatibility antigens in hematopoietic stem cell transplantation. Am J Transplant 2008; 8: 2149-2157.

9 Gratwohl A, Hermans J, Niederwieser D, Biezen A, Houwelingen HC, Apperley J. Female donors influence transplant-related mortality and relapse incidence in male recipients of sibling blood and marrow transplants. Hematol J 2001; 2: 363-370.

10 Toda I, Tsubota K. Practical double vital staining for ocular surface evaluation. Cornea 1993; 12: 366-367.

11 Tsubota K, Toda I, Yagi Y, Ogawa Y, Ono M, Yoshino K. Three different types of dry eye syndrome. Cornea 1994; 13(3): 202-209.
12 Shimmura S, Ono M, Shinozaki K, Toda I, Takamura E, Mashima $\mathrm{Y}$ et al. Sodium hyaluronate eyedrops in the treatment of dry eyes. Br J Ophthalmol 1995; 79: 1007-1011.

13 Lemp MA. Report of the National Eye Institute/Industry workshop on Clinical Trials in Dry Eyes. CLAO J 1995; 21: 221-232.

14 van Bijsterveld OP. Diagnostic tests in the Sicca syndrome. Arch Ophthalmol 1969; 82: 10-14.

15 Ban Y, Ogawa Y, Goto E, Uchino M, Terauchi N, Seki M et al. Tear function and lipid layer alterations in dry eye patients with chronic graft-vs-host disease. Eye 2009; 23: 202-208.

16 Tsubota K. The importance of the Schirmer test with nasal stimulation. Am J Ophthalmol 1991; 111: 106-108.

17 The definition and classification of dry eye disease: report of the Definition and Classification Subcommittee of the International Dry Eye WorkShop (2007). Ocul Surf 2007; 5: 75-92.

18 Scrucca L, Santucci A, Aversa F. Regression modeling of competing risk using $\mathrm{R}$ : an in depth guide for clinicians. Bone Marrow Transplant 2010; 45: 1388-1395.

19 Scrucca L, Santucci A, Aversa F. Competing risk analysis using R: an easy guide for clinicians. Bone Marrow Transplant 2007; 40: 381-387.

20 Copelan EA. Hematopoietic stem-cell transplantation. $N$ Engl J Med 2006; 354: 1813-1826.

21 Miklos DB, Kim HT, Zorn E, Hochberg EP, Guo L, Mattes-Ritz A et al. Antibody response to DBY minor histocompatibility antigen is induced after allogeneic stem cell transplantation and in healthy female donors. Blood 2004; 103: 353-359.

22 Miklos DB, Kim HT, Miller KH, Guo L, Zorn E, Lee SJ et al. Antibody responses to $\mathrm{H}-\mathrm{Y}$ minor histocompatibility antigens correlate with chronic graft-versus-host disease and disease remission. Blood 2005; 105: 2973-2978.

23 Flowers ME, Pepe MS, Longton G, Doney KC, Monroe D, Witherspoon RP et al. Previous donor pregnancy as a risk factor for acute graft-versus-host disease in patients with aplastic anaemia treated by allogeneic marrow transplantation. Br J Haematol 1990; 74(4): 492-496.

24 Kollman C, Howe CWS, Anasetti C, Antin JH, Davies SM, Filipovich $\mathrm{AH}$ et al. Donor characteristics as risk factors in recipients after transplantation of bone marrow from unrelated donors: the effect of donor age. Blood 2001; 98: 2043-2051.

25 Carlens S, Ringden O, Remberger M, Lonnqvist B, Hagglund $\mathrm{H}$, Klaesson $\mathrm{S}$ et al. Risk factors for chronic graftversus-host disease after bone marrow transplantation: a retrospective single centre analysis. Bone Marrow Transplant 1998; 22: 755-761.

26 Przepiorka D, Smith TL, Folloder J, Khouri I, Ueno NT, Mehra $\mathrm{R}$ et al. Risk factors for acute graft-versus-host disease after allogeneic blood stem cell transplantation. Blood 1999; 94: 1465-1470. 